\title{
A REDUCED COMPLEXITY AND AN EFFICIENT CHANNEL ESTIMATION FOR OFDM
}

\author{
Lokesh A. Panchal \\ ${ }^{1} B E, M \cdot E(E C)$
}

\begin{abstract}
During the last few years, the progress in wireless communication is widely increasing to mitigate the ever increasing demand of higher data rates. OFDM (Orthogonal Frequency Division Multiplexing) techniques using more densely packed carriers, thus achieving higher data rates using similar channels.

In this paper, the estimation is based on the minimum mean square error (MMSE) estimator and the least square (LS) estimator. I derive the MMSE and LS estimators architecture and investigate their performances. I proved that the MMSE estimator performance is better but computational complexity is high, contrary the LS estimator has low complexity but poor performance. For reducing complexity I proposed two different solutions which are the Latest Least Square (LLS) estimator and the modified MMSE estimator. I evaluate estimator's performance on basis of mean square error and symbol error rate for modulation systems.
\end{abstract}

Keywords: OFDM, LS, MMSE, LLS and Modified MMSE, SNR

\section{INTRODUCTION}

The research on wireless communication systems with high data rate, high spectrum efficiency and reliable performance is a hotspot. This OFDM is an efficient high data rate transmission technique for wireless communication.

OFDM presents advantages of high spectrum efficiency, simple and an efficient implementation by using DFT and IDFT, mitigation of inter-symbol interference (ISI) by inserting cyclic prefix and robustness to frequency selective fading channel. The received signal is usually distorted by the channel characteristics. To recover the transmitted bits, the channel effect must be estimated by estimators and received in the receiver section. There are two major issues when in designing channel estimators for wireless OFDM systems. The first issue is the arranging in the pilot or reference signal for transmitters and receivers section. The second issue is the designing of estimation with both low complexity and high performance. To maintain high data rates and low bit error rates in OFDM systems, the estimation should have low complexity and high accuracy.

Both estimators LS and MMSE have some own drawbacks. However the MMSE estimator performance is better but computational complexity is high, contrary the LS estimator has high mean-square error but its computational complexity is very low. For reducing computational complexity and improve performance, I proposed two channel estimation approaches.

\section{SYSTEM DIAGRAM}

As shown in below figure, the input serial binary data stream will be entered by a data scrambler first and then channel coding is applied to the input data to improve the
BER (bit error rate) performance of the system. The encoded data stream is further interleaved to reduce the burst symbol error rate. Various base modulation modes such as BPSK (binary phase shift keying) and QAM are adaptively used to boost the data rate. The transmission of data frames can change by modulation mode[1].

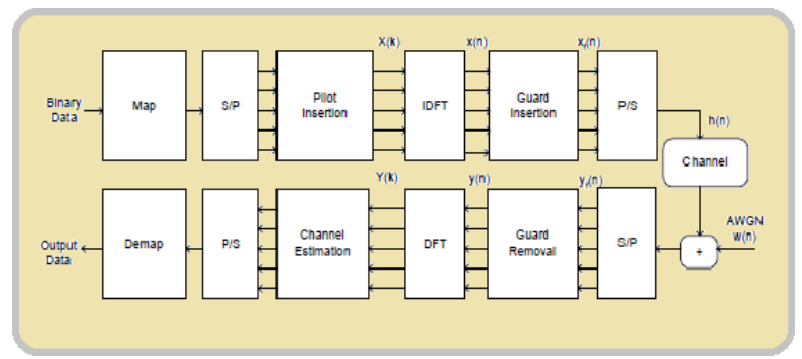

Fig.1: Basic OFDM system

At the receiver, after passing to discrete domain through $\mathrm{A} / \mathrm{D}$ and low pass filter, guard time is removed and $y(n)$ is sent to DFT block. Following DFT block, the pilot signals are extracted and the estimated channel $H_{e}(k)$ for the data sub-channels is obtained in channel estimation block. After the estimation of the transmitted data by[2]:

$$
\widehat{H} \mathrm{LS}[\mathrm{k}]=\frac{Y[k]}{X[k]},(\mathrm{k}=0,1,2, \ldots, \mathrm{N}-1) 1.1
$$

\section{PROPOSED CHANNEL ESTIMATION}

There are basically two proposed schemes for channel estimation, which are based on less complexity related with calculation and an efficient. Both proposed techniques are described below: 


\subsection{LLS Channel Estimation}

Our first modified version is based on LS estimator. The LS estimator characteristic is high mean square error. For improving the performance and to reduce the computation complexity, we proposed the following LLS estimator.

From above equation 4.3,

$$
H_{L S}=\mathrm{h}+\mathrm{n} 1.2
$$

Where $\mathrm{h}$ impulse response and $\mathrm{n}$ is the noise

$$
\mathrm{h}=\mathrm{F} \cdot \mathrm{g}
$$

Where $\mathrm{F}$ is the DFT matrix and $\mathrm{g}$ is the channel Impulse response

From equation (1.2), the LS estimator consists of channel transfer function plus some noise. Due to noise part the LS estimator gives the poor performance. To improve the estimation performance, we have to remove the noise from the original signal by estimating the channel. The LS estimation is noisy observation of the channel attenuation which can be smoother using some auto-correlation operation with the channel attenuation $H_{L S}$. If the channel transfer function is $\boldsymbol{h}$, the received signal $\boldsymbol{y}$ and the transmitted symbol $\boldsymbol{x}$, then the LLS channel estimator will be:

$$
H_{L L S}=W_{x} \cdot H_{L S} 1.3
$$

Here,

$$
W_{x}=R_{h h} / R_{h h}+\frac{\sigma_{n}^{2}}{x \cdot x^{H}}
$$

Where $R_{h h}=\mathrm{E}\left\{\mathrm{h} \cdot h^{H}\right\}$

Where, $R_{h h}$ is the auto-covariance matrix of $\boldsymbol{h}$. The weighting matrix $W_{x}$ of size $N \times N$ depends on the transmitted signal $\boldsymbol{x}$. As a step towards the low-complexity estimators we want to find a weighting matrix which does not depend on the transmitted signal $\boldsymbol{x}$. The weighting matrix can be obtained from the auto-covariance matrix of $\boldsymbol{h}$ and auto-correlation of transmitted signal $\boldsymbol{x}$. Assuming here the transmitted signal $\boldsymbol{x}$ to be stochastic with independently and uniformly distributed constellation signals. In this case the auto-covariance matrix of noise becomes

$$
R_{n n}=\frac{\alpha}{S N R} \mathrm{I} 1.4
$$

Where, $\alpha$ is constellation factor and $\mathrm{E}\left\{X_{i}^{2}\right\} \cdot E\left\{\frac{1}{X_{i}^{2}}\right\}$ is the mathematical expression of $\alpha$. The value of $\alpha$ is $17 / 6$ for 16 QAM. SNR is a per-symbol signal-to-noise ratio equal to $\frac{\mathrm{E}\left\{X_{i}^{2}\right\}}{\delta_{n}^{2}}$. Then the LLS estimator becomes

$$
H_{L L S}=W_{\text {modified }} \cdot H_{L S} 1.5
$$

Where,

$$
W_{\text {modified }}=\frac{R_{h h}}{R_{h h}+R_{n n}}
$$

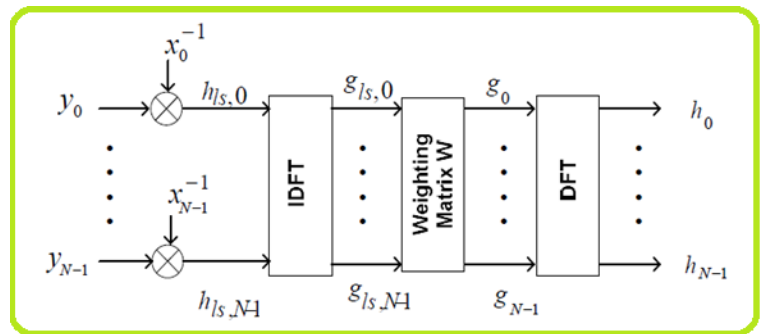

Fig.2: Block Diagram of LLS Estimation

Above shows the diagram of $H_{L L S}$ estimation, $x_{n}$ are the input signals, $y_{n}$ are the output samples, $g_{n}$ is the channel impulse response in time domain and $h_{n}$ is the channel transfer function in the frequency domain.

\subsection{Modified MMSE}

The modified estimator is based on MMSE estimator. Most of the channel energy $g$ is contained in or near to the first $(\mathrm{L}+1)$ samples, where $\mathrm{L}$ is $\left[\frac{\mathrm{Tg}}{\mathrm{Ts}}\right] \mathrm{N}, \mathrm{Tg}$ is the cyclic extension of time length, Ts is sampling interval and $\mathrm{N}$ is the DFT size. Therefore to modify the estimator we consider only the significant energy samples that are the upper left corner of auto-covariance matrix, $\mathrm{R}_{\mathrm{gg}}$. From the IEEE std. 802.11 and IEEE std 802.16, $\left[\frac{\mathrm{Tg}}{\mathrm{Ts}}\right] \mathrm{N}$ should be chosen among $\{1 / 32$, $1 / 16,1 / 8\}$. In Figure 5 , the significant energy level is 8 . So $\left[\frac{\mathrm{Tg}}{\mathrm{Ts}}\right]=1 / 8$ and $\mathrm{L}=1 / 8 \times 64=8$. So, the significant energy consists of 1 to 8 sample and remaining samples are noise of low SNR. To reduce the complexity we consider only the significant energy samples.

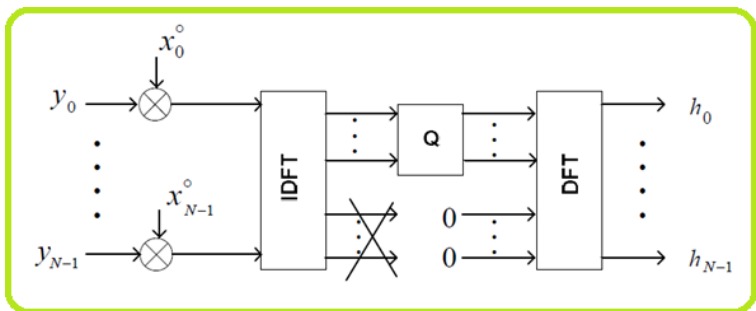

Fig.3: Modified MMSE channel Estimation

Figure 9, shows the general structure of Modified MMSE estimator. Where $\mathrm{x}_{\mathrm{n}}$ is the input signal, $\mathrm{y}_{\mathrm{n}}$ is output signal, $\mathrm{Q}$ is frequency response in time domain and $h_{n}$ is the transfer function, all these variables are range from 0 to $\mathrm{N}$-th sample. In modified $\mathrm{Q}_{\mathrm{MMSE}}$ estimator, we consider only the significant energy samples that samples are transmit the data signal and remaining samples transmit null signal. In MMSE-4 estimator, first three samples send data signal and remaining samples send null signal. By implement the same approach, MMSE-6, MMSE-8 estimators data signal are consists of four, five, six and eight samples respectively and the rest of data bit information is set to null signal. 
The goal of the simulation is efficiently estimate the channel and then validation of the proposed method. The simulation scenarios enable analysis of different channel estimator performance to find the optimal channel estimator with low complexity. The significant energy level is one of the major factors to determine estimator performance.

\section{SIMULATION RESULT AND DISCUSSIONS}

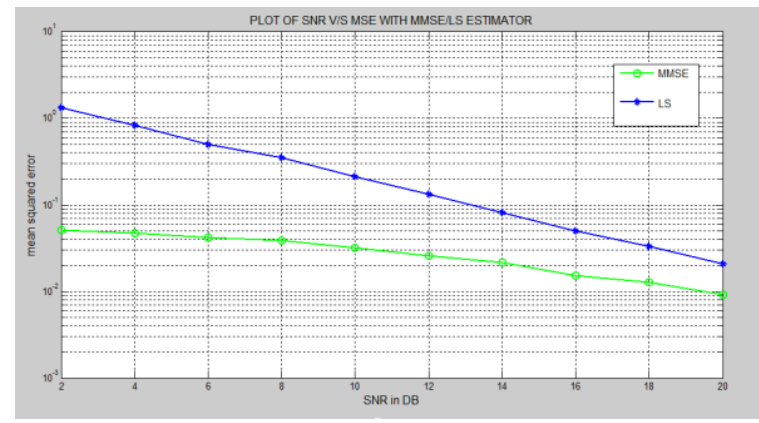

Fig.4: MMSE and LS estimator performance comparison based on characteristics of MSE Vs SNR

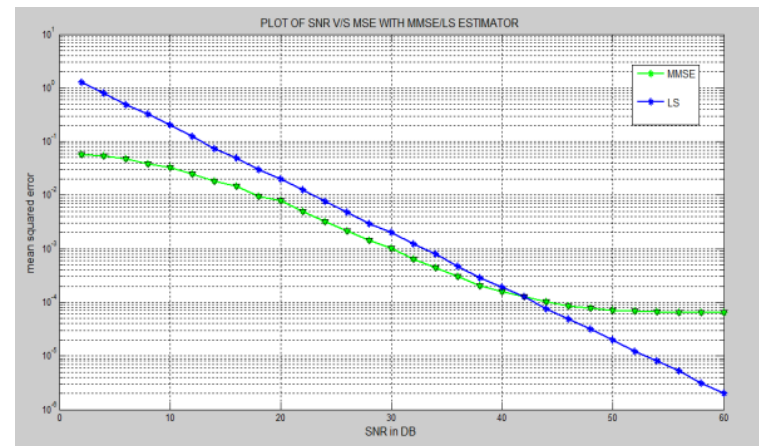

Fig.5: MMSE and LS estimator performance comparison based on characteristics of MSE Vs SNR (for higher range of SNR)

Above figures 4 and 5 show the mean square error versus SNR curve for LS and MMSE. For SNR range from $2 \mathrm{~dB}$ to $20 \mathrm{~dB}$, the MMSE estimator mean square error range is $10^{-3}$ to $10^{-1}$ whereas the LS estimator mean square error range is $10^{-3}$ to $10^{0}$. While SNR range increases from $4 \mathrm{~dB}$ to $60 \mathrm{~dB}$, the MMSE estimator mean square error range is $10^{-4}$ to $10^{-1}$, whereas the LS estimator mean square error range is $10^{-6}$ to $10^{0}$. LS and MMSE, the both of estimators give lower mean square error for higher range of SNR.

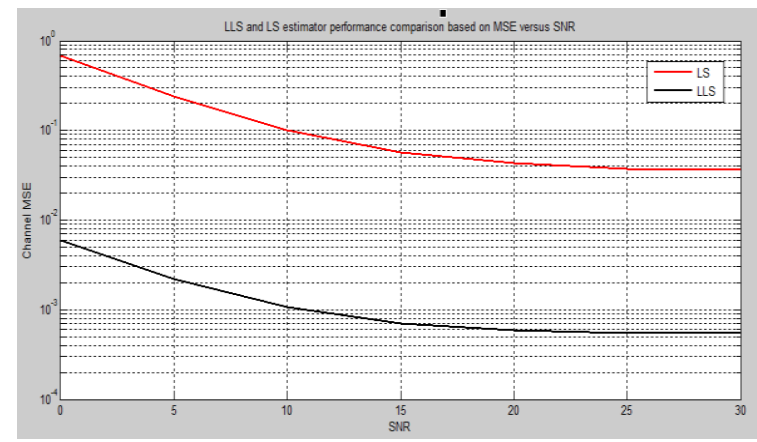

Fig.6: LS and LLS estimator performance comparison based on MSE Vs SNR (Lower SNR)
Above figure 6 shows the characteristics of MSE versus SNR for the LS and LLS estimators respectively. The LLS estimator performance is better than LS.

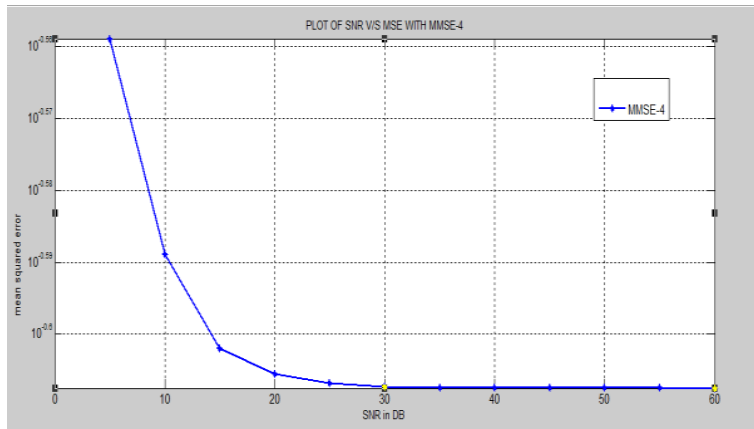

Fig.7: Estimator performance for MMSE-4 based on MSE versus SNR

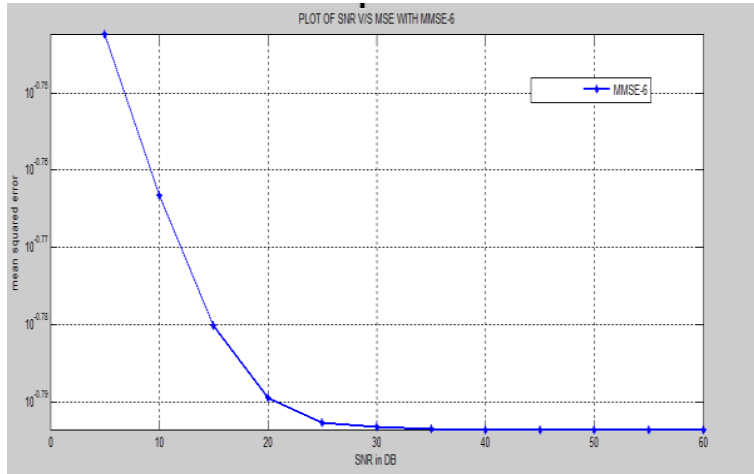

Fig.8: Estimator performance for MMSE-6 based on MSE versus SNR

Above figures 7 and 8 represent the performance analysis of MMSE-4 and MMSE-6 for the SNR range from $5 \mathrm{~dB}$ to 60 $\mathrm{dB}$. The MMSE-4 estimator MSE is from $10^{-0.60}$ to $10^{-0.56}$ and for MMSE- 6 the range is $10^{-0.79}$ to $10^{-0.74}$. The modified MMSE estimator's performance is mainly depend on the significant energy samples. The MMSE-6 estimator gives the lower MSE compare than the MMSE-4 estimator. The significant energy level for the MMSE-4 is 0 to 3 samples, whereas the MMSE-6 is 0 to 5 samples. From 30 $\mathrm{dB}$ SNR, the MMSE-4 estimator MSE is less than 0.001, whereas the MMSE-6 estimator is less than 0.002. In higher range of SNR, both of these estimators' give the lower MSE.

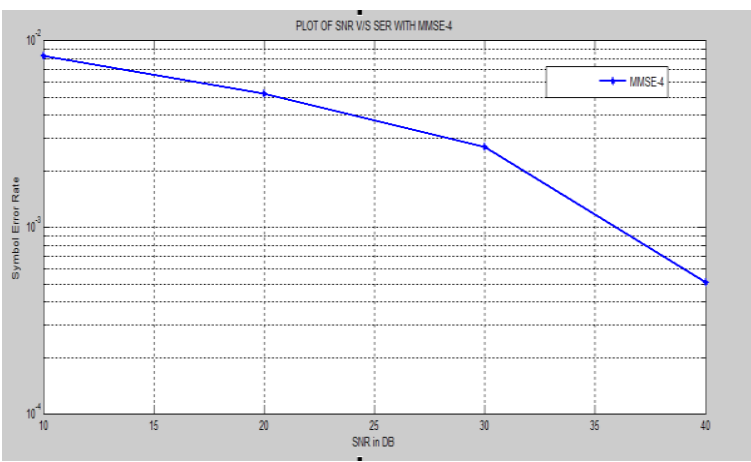

Fig.9: Estimator performance for MMSE-4 based on SER versus SNR 


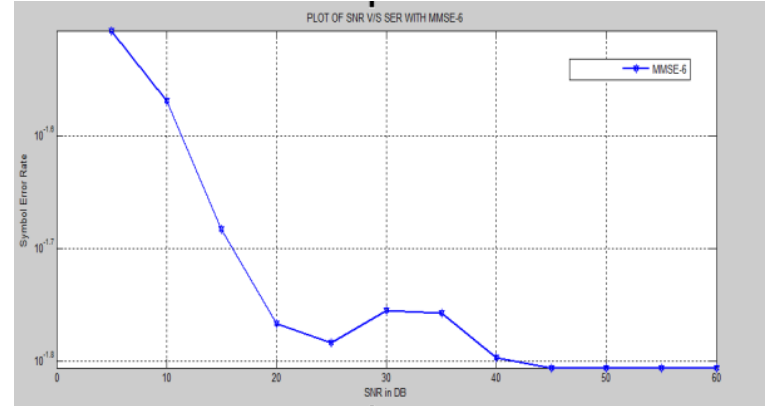

Fig.10: Estimator performance for MMSE-6 based on SER versus SNR

Figures 9 and 10 provide the performance analysis for MMSE-4 and MMSE-6. For the SNR range from $10 \mathrm{db}$ to $60 \mathrm{~dB}$, MMSE-6 estimator SER is $10^{-0.35}$ to $10^{-0.35}$ whereas SER of MMSE -6estimator is $10^{-1.8}$ to $10^{-1.5}$. So the higher number of significant energy sample can be reduce the SER range. The SER curves have major declined from upward to downward direction in all these estimators' in the interval of 10-25 dB SNR range.

\section{CONCLUSIONS}

In wireless environment there may noise or interference may include with desired signal. So here requiring channel estimation.

In the channel estimation in OFDM here in this paper I found solution for existed problems based on simulation result in Channel estimation techniques. Basic purpose of this proposed estimation is to give better performance with low complexity and an efficient than existed techniques.

\section{REFERENCES}

[1]. Sinem Coleri, Mustafa Ergen, Anuj Puri, Ahmad Bahai, "Channel Estimation Techniques Based on Pilot Arrangement in OFDM Systems" IEEE transactions on broadcasting, vol. 48, no.- 3, september 2002.

[2]. Sajjad Ahmed Ghauri, SherazAlam, M. Farhan Sohail, AsadAli, Faizan Saleem "Implementation of ofdm and channel estimation using LS and MMSE estimators", IJCER, Vol.2, Feb.2013.

[3]. R.S.Ganesh, Dr.J.JayaKumari, “A Survey on Channel Estimation Techniques in MIMO-OFDM Mobile Communication Systems", IJSER, Volume 4, Issue 5, May2013

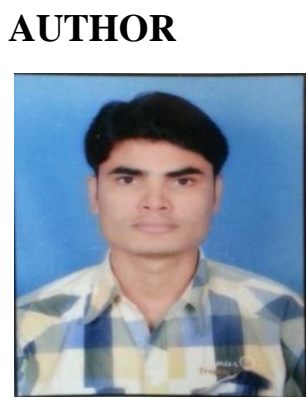

Lokesh A. Panchal was born on March 28th, 1990, India. He graduated in Electronics and Communications Engineering, from Sankalchand Patel College fo Engg., Visnagar, India in June,2011.He has also telecom fieldExperience for a year. In 2014 , he is pursuing the master's degree of research in telecommunication in SITER, Ahmedabad, India. His research area is channel estimation in OFDM system. 